\title{
Cognitive outcome after unilateral pallidal stimulation in Parkinson's disease
}

Guy Vingerhoets, Chris van der Linden, Engelien Lannoo, Veerle Vandewalle, Jacques Caemaert, Mercedes Wolters, Dirk Van den Abbeele

\begin{abstract}
Objectives-Chronic high frequency electrostimulation of the globus pallidus internus mimics pallidotomy and improves clinical symptoms in Parkinson's disease. The aim of this study was to investigate the cognitive consequences of unilateral deep brain stimulation.

Methods-Twenty non-demented patients with Parkinson's disease (age range 38-70 years) were neuropsychologically assessed 2 months before and 3 months after unilateral pallidal stimulation. The cognitive assessment included measures of memory, spatial behaviour, and executive and psychomotor function. In addition to group analysis of cognitive change, a cognitive impairment index (CII) was calculated for each individual patient representing the percentage of cognitive measures that fell more than 1 SD below the mean of a corresponding normative sample.
\end{abstract}

Results-Neurological assessment with the Hoehn and Yahr scale and the unified Parkinson's disease rating scale disclosed a significant postoperative reduction in average clinical Parkinson's disease symptomatology $\quad(p<0.001)$. Repeated measures multivariate analysis of variance (using right/left side of stimulation as a between subjects factor) showed no significant postoperative change in cognitive performance for the total patient group (main effect of operation). The side of stimulation did not show a significant differential effect on cognitive performance (main effect of lateralisation). There was no significant operation by lateralisation interaction effect. Although the patients experienced significant motor symptom relief after pallidal stimulation, they remained mildly depressed after surgery. Analysis of the individual CII changes showed a postoperative cognitive decline in $30 \%$ of the patients. These patients were significantly older and took higher preoperative doses of levodopa than patients showing no change or a postoperative cognitive improvement. Conclusions-Left or right pallidal stimulation for the relief of motor symptoms in Parkinson's disease seems relatively safe, although older patients and patients needing high preoperative doses of levodopa seem to be more vulnerable for cognitive decline after deep brain stimulation. (F Neurol Neurosurg Psychiatry 1999;66:297-304)
Keywords: Parkinson's disease; pallidal stimulation; neuropsychology; pallidotomy

Pharmacological treatment of Parkinson's disease provides symptomatic benefit, but fails to maintain long term symptom relief. ${ }^{1}$ Chronic antiparkinson treatment may eventually lead to marked "on-off" fluctuations or loss of effect. ${ }^{2} 3$ As a result, surgical treatment in patients with advanced Parkinson's disease has seen a renewed interest.

Pallidotomy under local anaesthesia has significantly reduced both the akinetic and hyperkinetic symptoms of Parkinson's disease although requiring most patients to maintain preoperative levodopa doses. ${ }^{4-7}$ It is assumed that nigral-striatal dopaminergic deficiency, the characteristic biochemical disturbance of Parkinson's disease, causes an inhibitory hyperactivity in the internal globus pallidus with resultant thalamocortical hypoactivity, inhibiting the initiation of movement and causing akinesia, rigidity, and tremor. ${ }^{8}$ Reduction of the inhibitory effect by lesioning the posteroventral areas of the human globus pallidus internus (GPi) that project to the ventrolateral thalamus increases movement related activity in motor association cortex and improves both akinesia, tremor, and rigidity, and the levodopa induced dyskinesias. $^{9-11}$

Functional pallidotomy is also achieved by chronic high frequency electrostimulation of the GPi. The advantage of his technique lies with its non-destructive and reversible nature. After pallidal stimulation marked postoperative improvement of the debilitating motor symptoms as evaluated by disease rating scales has been found. ${ }^{12-15}$

Although the reduction of motor symptoms after (functional) unilateral pallidotomy seems significant, the evaluation of cognitive outcome is warranted. Firstly, evidence has accumulated that the basal ganglia, of which the GPi is the major output centre, also contribute to nonmotor cognitive and emotional functions, especially through the frontostriatal pathways. For example, pallidotomy has been associated with modulations in brain metabolism in (dorsolateral) frontal cortical regions, ${ }^{9} 1617$ although this has been disputed. ${ }^{18}$ Secondly, animal experiments have disclosed a relation between pallidal stimulation and hippocampal activity presumed to participate in the control of motor behaviour. ${ }^{19}{ }^{20}$ In humans the hippocampus is critical for the acquisition of new factual knowledge (learning) and the (dorsolateral) frontal region has been linked with working memory and problem solving. ${ }^{21}$ Thirdly, it 
Table 1 Preoperative demographic and neurological data of the patients with Parkinson's disease (PD)

\begin{tabular}{|c|c|c|c|c|c|c|c|c|c|c|}
\hline $\begin{array}{l}\text { Patient } \\
\text { No }\end{array}$ & Sex & $\begin{array}{l}\text { Age } \\
(y)\end{array}$ & $\begin{array}{l}\text { Education } \\
(y)\end{array}$ & $\begin{array}{l}\text { Estimated } \\
I Q\end{array}$ & $\begin{array}{l}\text { Age of } \\
\text { Disease } \\
\text { Onset }\end{array}$ & $\begin{array}{l}\text { PD } \\
\text { Duration } \\
\text { (y) }\end{array}$ & $\begin{array}{l}\text { Most } \\
\text { affected } \\
\text { side }\end{array}$ & $\begin{array}{l}\text { Levodopa } \\
\text { dosage } \\
\text { mg/day }\end{array}$ & $\begin{array}{l}\text { Years of } \\
\text { levodopa } \\
\text { treatment }\end{array}$ & $\begin{array}{l}\text { Other } \\
\text { Medication }\end{array}$ \\
\hline 1 & M & 39 & 15 & 109 & 15 & 24 & Right & 500 & 9 & $\mathrm{P}$ \\
\hline 2 & M & 60 & 9 & 86 & 56 & 5 & Right & 400 & 5 & B \\
\hline 3 & M & 38 & 8 & 81 & 30 & 8 & Right & 425 & 8 & B \\
\hline 4 & $\mathrm{~F}$ & 58 & 10 & 89 & 48 & 10 & Right & 1200 & 3 & B \\
\hline 5 & $\mathrm{M}$ & 44 & 12 & 109 & 32 & 12 & Left & 1100 & 8 & $\mathrm{~B} / \mathrm{AC}$ \\
\hline 6 & $\mathrm{M}$ & 49 & 13 & 116 & 40 & 10 & Right & 650 & 10 & $\mathrm{~B} / \mathrm{P}$ \\
\hline 7 & M & 44 & 12 & 111 & 39 & 6 & Left & 675 & 5 & $\mathrm{~B} / \mathrm{AC}$ \\
\hline 8 & M & 67 & 12 & 99 & 53 & 15 & Right & 1000 & 15 & $\mathrm{~B} / \mathrm{P} / \mathrm{AD}$ \\
\hline 9 & M & 67 & 10 & 95 & 58 & 9 & Right & 1050 & 9 & $\mathrm{~B} / \mathrm{P}$ \\
\hline 10 & M & 45 & 11 & 105 & 38 & 6 & Left & 800 & 6 & B \\
\hline 11 & M & 70 & 16 & 115 & 55 & 16 & Right & 850 & 16 & B \\
\hline 12 & $\mathrm{~F}$ & 47 & 16 & 115 & 37 & 10 & Left & 700 & 7 & $\mathrm{P}$ \\
\hline 13 & $\mathrm{~F}$ & 57 & 10 & 104 & 52 & 5 & Left & 875 & 5 & $\mathrm{P}$ \\
\hline 14 & M & 62 & 12 & 93 & 58 & 4 & Right & 1200 & 4 & $\mathrm{P}$ \\
\hline 15 & M & 57 & 10 & 104 & 47 & 10 & Right & 925 & 7 & B \\
\hline 16 & M & 60 & 10 & 85 & 52 & 9 & Right & 800 & 9 & B \\
\hline 17 & $\mathrm{~F}$ & 46 & 10 & 104 & 43 & 3 & Left & 300 & 3 & $\mathrm{~B} / \mathrm{P}$ \\
\hline 18 & M & 62 & 8 & 102 & 42 & 20 & Right & 550 & 20 & B/P/AC \\
\hline 19 & $\mathrm{~F}$ & 62 & 11 & 78 & 54 & 9 & Left & 800 & 9 & $\mathrm{P}$ \\
\hline 20 & M & 64 & 12 & 76 & 64 & 1 & Right & 300 & 1 & - \\
\hline Mean & & 55 & 11 & 99 & 45 & 9.6 & & 755 & 8.0 & \\
\hline SD & & 9.9 & 2.3 & 12.7 & 11.6 & 5.7 & & 280 & 4.7 & \\
\hline
\end{tabular}

$\mathrm{B}=$ Bromocriptine $\mathrm{P}=$ pergolide $\mathrm{AC}=$ anticholinergic drugs $\mathrm{AD}=$ antidepressant drugs.

remains uncertain whether the asymmetric dopaminergic activity that presumably underlies asymmetry of motor symptoms in certain patients with Parkinson's disease relates to possible lateralisation of cognitive dysfunction. $^{22-24}$ The differential effect of left versus right deep brain stimulation on cognition in patients with asymmetric Parkinson's disease remains to be determined.

The group of Baron and Green ${ }^{725}$ evaluated the neuropsychological outcome after unilateral pallidotomy in 13 patients with Parkinson's disease and concluded that ventrolateral lesions targeting the sensorimotor GPi region were not associated with pronounced postoperative cognitive change. Soukop et al ${ }^{26}$ prospectively investigated 14 patients with Parkinson's disease on cognitive changes after unilateral posteroventral pallidotomy and also found no significant deterioration in specific cognitive abilities as a result of the procedure. These authors found a significant improvement in motor coordination speed for both contralateral and ipsilateral upper limbs. By contrast, the group of Lang and Saint-Cyr ${ }^{27} 28$ documented discrete neuropsychological and behavioural changes as a consequence of GPi pallidotomy in a larger sample of patients. Attentional capacity often improved postoperatively. Transient impairment in phonemic verbal fluency and serial list learning, and more long term impairments in category fluency were found after left hemispheric lesions. Transient impairments in the encoding of a complex figure were seen after right sided lesions.

In this study we report on the cognitive outcome after unilateral pallidal stimulation. We evaluated the postoperative changes in 20 patients undergoing unilateral stimulation of the GPi. The study aimed to investigate how cognition might be affected in Parkinson's disease after left or right pallidal stimulation. Both group effects and individual outcome data are presented.

\section{Methods}

SUBJECTS

Patients were considered for unilateral pallidal stimulation because of (1) bradykinesia and rigidity predominant Parkinson's disease; (2) response fluctuations with "off" parkinsonism and "on" dyskinesias; (3) or both. The target was determined contralateral to the more affected side. The following exclusion criteria for idiopathic Parkinson's disease were employed: (1) parkinsonism associated with a history of known causative factors such as encephalitis or neuroleptic treatment; (2) the presence of dementia, supranuclear gaze abnormalities, or ataxia; (3) no convincing response to levodopa. Further exclusion criteria for the neuropsychological study were (1) the presence of medical, neurological (other than Parkinson's disease), or orthopaedic illness that would compromise neuropsychological assessment; (2) focal brain abnormalities on MRI; (3) a score <24 on the mini mental state examination (MMSE); (4) not a native speaker of the testing language (Flemish Dutch). Twenty of 23 consecutive idiopathic patients with Parkinson's disease (15 men and five women; mean age 55 (SD 9.9), mean years of education 11 (SD 2.3)) met these criteria and were included in the study. Of the three patients that were not included, two were French speaking and one patient had an MMSE score of 20 . Thirteen patients underwent stimulation of the left globus pallidus internus and seven patients had the electrode implanted in the right GPi. Demographic and neurological data for these patients are listed in table 1 .

\section{PROCEDURE}

Patients were neuropsychologically and neurologically assessed during the presurgical evaluation programme 2 months before surgery, and again 3 months after surgery. The assessment took place during the "on" phase of medication before surgery, and with the stimulator "on" after surgery. 
Neuropsychological assessment

The patients' cognitive status was assessed on four cognitive domains that have been reported to decline in patients with Parkinson's disease-namely, memory, visuomotor coordination, spatial behaviour, and executive functioning. ${ }^{29} 30$ We used 10 standardised neuropsychological tests out of which 12 cognitive measures were selected. The tests and retained measures are listed below.

Memory - (1) The Rey auditory verbal learning test (AVLT) assesses verbal memory. ${ }^{31}$ To reduce test-retest effects, we used randomised alternate versions before and after surgery. The measures retained are the total number of words immediately recalled over the first five trials and the delayed recall of the original list.

(2) The recognition form (motor free) of the Benton visual retention test (BVRT) assesses short term non-verbal memory. ${ }^{32}$ The number of correct answers is the measure taken.

Visuomotor coordination-(3) The Purdue pegboard test (PPT) measures finger and hand dexterity. ${ }^{31}$ The total number of pegs placed in 30 seconds with both hands simultaneously was measured. (4) Visual reaction time (VRT), requiring a simple motor reaction (pressing a lever with the index finger) to a simple visual stimulus, was measured with the Fepsy computer program..$^{33}$ The median of 30 trials for each hand was calculated.

Spatial behaviour-(5) The (motor free) subtests "number location" and (6) "cube analysis" of the visual object and space perception battery (VOSP) were used to evaluate visuospatial localisation and spatial reasoning respectively. ${ }^{34}$ The number of correct responses for each of the motor free subtests were recorded. (7) Money's standardised road map test for direction sense (MRMT) was used to assess spatial (left-right) orientation. ${ }^{31}$ When needed, the verbal answers of the patient were noted by the neuropsychologist. The number of correct responses was the measure taken.

Executive functioning - (8) The standardised Dutch version of the Stroop coloured word test (SCWT) measures divided attention and resistance to cognitive interference. ${ }^{31}{ }^{35}$ (9) Interference score-The controlled oral word association test (COWAT) assesses verbal fluency. ${ }^{31}$ The total number of words presented during three 1 minute orthographic categories was measured. (10) A computerised version of the Wisconsin card sorting test (WCST) was used to assess mental flexibility. ${ }^{36}$ When needed, the verbal answers of the patient were entered by the neuropsychologist. The measure taken was the number of categories achieved.

In addition to the neuropsychological battery, two other psychometric tests were administered. A Dutch version of the national adult reading test (NART) was used to estimate premorbid verbal intelligence. ${ }^{37} \mathrm{~A}$ Dutch version of the Beck depression inventory (BDI) was used to estimate the level of depression before and after surgery. ${ }^{38}$ Because the subjects are medical patients that have marked physical symptoms and had undergone neurosurgery, vegetative and somatic symptoms picked up by the BDI subscale that measures somatic performance complaints, might overestimate the severity of their depressions. ${ }^{38-41}$ For this reason, only the 13 item cognitive/affective subscale of the BDI was used to estimate the patients' level of depression (BDI-C/A).

\section{Neurological assessment}

Patients were neurologically rated by a neurologist (CVDL) according to the modified Hoehn and Yahr scale ${ }^{42}$ and the unified Parkinson's disease rating scale (UPDRS). ${ }^{43}$ The modified Hoehn and Yahr scale offers an estimate of the patient's stage (from stage 0: no signs of Parkinson's disease, to stage 5: wheelchair bound or bedridden unless aided). The UPDRS is a comprehensive symptoms and signs checklist that consists of four major subscales: mentation, behaviour, and mood (UPDRS-I, maximum score $=16$ ), activities of daily living (UPDRS-II, maximum score $=52$ ), motor examination (UPDRS-III, maximum score $=108)$, and complications of therapy (UPDRS-IV). The sum of the first three subscales provides a total UPDRS score (maximum score $=176$ ) that offers a general estimate of the severity of the patient's symptoms.

SURGICAL PROCEDURE

A stereotactic (Leksell G) frame was fixed to the skull under local anaesthesia. The target was chosen in the external segment of the internal globus pallidus (GPi) using modified Leksell coordinates: $4 \mathrm{~mm}$ anterior to the midcommisural point, $2 \mathrm{~mm}$ inferior to the intercommisural line, and $18-22 \mathrm{~mm}$ lateral to the AC-PC line. The target was defined using both CT and ventriculography. The angle of approach was chosen as perpendicular as possible to both coronary and sagittal planes, thereby avoiding the lateral ventricle. The target for the injection of the contrast medium for ventriculography was defined on CT in the frontal horn of the lateral ventricle close to the foramen of Monro ipsilateral to the target side. The stereotactic coordinates of the stimulation target as defined by CT were installed on the Leksell frame. After introducing a burr hole for the insertion of the stimulation electrode, the coordinates for ventriculography were installed on the frame. After incising the dura, a $2.1 \mathrm{~mm}$ catheter was inserted with the tip in the target for ventriculography. A total of $5 \mathrm{ml} \mathrm{CSF}$ was withdrawn and replaced by $3 \mathrm{ml}$ of contrast medium immediately followed by a lateral skull radiograph. This was repeated for a skull radiograph in the anterior-posterior direction. The invisible target was calculated by the Leksell localiser using the AC and PC as landmarks and the atlas coordinates of the GPi according to Schaltenbrand. The definite coordinates for electrostimulation were based on both CT and ventriculography measurements and installed on the stereotactic frame. Using the same cortical incision for ventriculography, a $1.8 \mathrm{~mm}$ rigid monopolar electrode (Radionics) was introduced while continuously monitoring the tissue impedance. At $7 \mathrm{~mm}$ above the presumed target test stimulation began 
with a frequency of $100 \mathrm{~Hz}$ and a stimulus duration of $1 \mathrm{~ms}$. The stimulus intensity was increased at increments of $0.5 \mathrm{~V}$ up to the desired response or untoward side effects. At each point of test stimulation the clinical response, if any, was evaluated by a neurologist blind to the presence of stimulation. The following clinical indices using the unified Parkinson's disease rating scale (UPDRS) were scored: tremor (if present) and rigidity in all four limbs, rapid finger movements, closing and opening of hands, and dorsiflexion and plantar flexion of the feet. If a clinical response was not present at the target or several points above the target, the electrode was withdrawn. A second target through different adjacent trajectories was selected $2 \mathrm{~mm}$ anterior, posterior, medial, or lateral to the initial target depending on the CT images used for calculating the coordinates. If necessary adjacent trajectories were introduced for target selection. After determination of the optimum stimulation site the monopolar electrode was replaced by the definitive quadripolar electrode (Medtronic, model 3387 DBS), with the electrode proximal to the most distal electrode at the target. Another test stimulation was performed to confirm the findings during test stimulation with the monopolar test electrode. At the level of the burr hole the electrode was fixed with methyl metacrylate and connected to an extension cable, which was externalised at around 7 $\mathrm{cm}$ from the burr hole. The extension cable was connected to an external pulse generator, which was used for test stimulation with selection of the optimal stimulation indices up to 6 days postoperatively. After determination of the optimum stimulation indices the electrode was connected under general anaesthesia to an internal pulse generator (Medtronic, model 7425) through an inner extension cable (Medtronic, model 7495). The pulse generator was implanted subcutaneously just inferior to the clavicle and ipsilateral to the electrode implantation site. None of the patients had intraoperative or postoperative complications.

\section{STATISTICAL ANALYSIS}

Wilcoxon signed ranks tests are used to assess the postoperative changes in Hoehn and Yahr and UPDRS scores.

To evaluate preoperative to postoperative group changes for the different cognitive measures, we used a repeated measures multivariate analysis of variance (MANOVA) to account for the multiple comparisons of interrelated cognitive variables. After comparing the groups that received left versus right pallidal stimulation on equality concerning demographic and medical variables, lateralisation of deep brain stimulation was added in the repeated measures MANOVA as a between subjects factor.

The problem with repeated assessments in group analysis is that the cognitive impairment of some patients is compensated and masked by the (normal) learning effects of repeated cognitive testing in other patients. If the data are reported as mean performances, the expected overall trend will be towards some improvement (learning effect). The most obvi- ous way to overcome the difficulty of comparing groups of subjects is to examine the change in individual performance over time and to use each patient as his or her own control.

To evaluate individual outcome, we calculated a cognitive impairment index (CII) for each patient's preoperative and postoperative cognitive performance representing the percentage of cognitive measures below the impairment criterion. The impairment criterion is defined as a performance that falls more than 1 SD below the mean of a corresponding normative sample (stratified by age, education, and sex). For the WCST and the visual reaction time, the impairment criteria were based on the normative data in the corresponding manuals. ${ }^{364}$ For the other tests, the impairment criteria were based on the results of a Flemish normative neuropsychological study. ${ }^{45}$ Although the construction of an impairment index is a worthwhile strategy to evaluate the general cognitive change of an individual patient, it is important to appreciate the limitations of this strategy. Detailed information is lost in a global "organicity" score that does not take the relative importance of the included variables into account. Similar CII scores can be obtained in patients with entirely different cognitive profiles. As a consequence, there is no way of knowing to what extent changes in the CII are clinically relevant. To compare the subgroups with and without postoperatively increased cognitive impairment on 12 important demographic and medical variables, we used Mann-Whitney tests for ratio and interval measurements and $\chi^{2}$ for dichotomous data. No corrections for multiple testing were included to enhance the possibility of detecting even minor differences between the groups. Although this strategy increases the risk of false positive findings (type I errors), it allows for an early detection of possible influential variables that can guide future surgical decisions.

\section{Results}

Table 2 shows the preoperative and postoperative modified Hoehn and Yahr stage, total UPDRS score, and cognitive impairment index for each patient. The Hoehn and Yahr stage was significantly lower after surgery (Wilcoxon signed ranks $=-3.64, \mathrm{p}<0.001$ ) and the total preoperative UPDRS score was also significantly decreased postoperatively (Wilcoxon signed ranks $=-3.83, \mathrm{p}<0.001)$. These changes indicate a significant postoperative reduction in average clinical Parkinson's disease symptomatology.

Compared with normative data, the patients with Parkinson's disease showed a mean preoperative cognitive impairment ratio of 36.2 (SD 15.3)\%. The average postoperative cognitive impairment measured 35.4 (SD 20.0)\%. This measure was not significantly different from the preoperative impairment. Pearson correlations showed no significant relation between the degree of preoperative and postoperative cognitive impairment and the patients' estimated intelligence. The preoperative cognitive impairment index showed no significant 
Table 2 Preoperative and postoperative modified Hoehn and Yahr stage, total UPDRS score, and cognitive impairment index (CII)

\begin{tabular}{|c|c|c|c|c|c|c|c|c|}
\hline Patient No & Surgery & $\begin{array}{l}\text { Preop Hoehn } \\
\text { and Yahr }\end{array}$ & $\begin{array}{l}\text { Postop } \\
\text { Hoehn and } \\
\text { Yahr }\end{array}$ & $\begin{array}{l}\text { Preop } \\
\text { UPDRS } \\
\text { score }\end{array}$ & $\begin{array}{l}\text { Postop } \\
\text { UPDRS } \\
\text { score }\end{array}$ & Preop CII (\%) & Postop CII (\%) & $\begin{array}{l}\text { Trend in } \\
\text { cognitive } \\
\text { change }^{1}\end{array}$ \\
\hline 1 & Left & 1.0 & 0.0 & 15 & 5 & 60.0 & 50.0 & $\uparrow$ \\
\hline 2 & Left & 1.5 & 1.0 & 40 & 10 & 33.3 & 25.0 & $\uparrow$ \\
\hline 3 & Left & 4.0 & 0.0 & 42 & 8 & 62.5 & 58.3 & $\uparrow$ \\
\hline 4 & Left & 3.0 & 2.5 & 39 & 21 & 40.0 & 58.3 & $\downarrow$ \\
\hline 5 & Right & 1.5 & 1.0 & 28 & 18 & 36.4 & 8.3 & $\uparrow$ \\
\hline 6 & Left & 4.0 & 1.0 & 40 & 29 & 33.3 & 33.3 & $\leftrightarrow$ \\
\hline 7 & Right & 2.0 & 2.0 & 24 & 5 & 25.0 & 8.3 & $\uparrow$ \\
\hline 8 & Left & 4.0 & 3.0 & 79 & 46 & 50.0 & 58.3 & $\downarrow$ \\
\hline 9 & Left & 3.0 & 1.5 & 43 & 34 & 50.0 & 66.7 & $\downarrow$ \\
\hline 10 & Right & 4.0 & 3.0 & 79 & 29 & 16.7 & 16.7 & $\leftrightarrow$ \\
\hline 11 & Left & 3.0 & 3.0 & 19 & 19 & 33.3 & 33.3 & $\leftrightarrow$ \\
\hline 12 & Right & 1.0 & 1.0 & 14 & 2 & 25.0 & 16.7 & $\uparrow$ \\
\hline 13 & Right & 4.0 & 3.0 & 31 & 18 & 41.7 & 41.7 & $\leftrightarrow$ \\
\hline 14 & Left & 3.0 & 2.5 & 47 & 32 & 50.0 & 66.7 & $\downarrow$ \\
\hline 15 & Left & 3.0 & 1.0 & 20 & 13 & 25.0 & 41.7 & $\downarrow$ \\
\hline 16 & Left & 3.0 & 1.0 & 11 & 8 & 25.0 & 16.7 & $\uparrow$ \\
\hline 17 & Right & 2.5 & 1.0 & 21 & 8 & 8.3 & 8.3 & $\leftrightarrow$ \\
\hline 18 & Left & 2.5 & 1.0 & 9 & 4 & 16.7 & 25.0 & $\downarrow$ \\
\hline 19 & Right & 3.0 & 2.0 & 43 & 30 & 58.3 & 50.0 & $\uparrow$ \\
\hline 20 & Left & 1.5 & 1.0 & 21 & 6 & 33.3 & 25.0 & $\uparrow$ \\
\hline Mean (SD) & & $2.7(1.0)$ & $1.6(1.0)$ & $33(20)$ & $17(12)$ & $36.2(15.3)$ & $35.4(20.0)$ & \\
\hline
\end{tabular}

$\uparrow=$ Postoperative cognitive improvement; $\leftrightarrow=$ no cognitive change; $\downarrow=$ postoperative cognitive impairment.

correlation with preoperative Hoehn and Yahr stage and UPDRS score. Postoperatively, the cognitive impairment index correlated moderately with the postoperative total UPDRS score $(r=0.55, \mathrm{p}<0.05)$.

Table 2 indicates that six patients $(30 \%)$ showed a postoperative decline in general cognitive performance. The mean increase in cognitive impairment of this subgroup measured $14.2 \%$ (SD $4.6 \%$ ) indicating an average new deficit score on one or two of the 12 cognitive measures. We compared the group of patients who showed a decline in postoperative performance with the group of patients that showed either no change ( $n=5)$ or an improvement $(n=9)$ in cognitive performance on 12 important demographic and medical variables (using Mann-Whitney tests for ratio and interval measurements and $\chi^{2}$ for dichotomous data). Both groups showed no differences for the variables of sex, years of formal education, preoperative cognitive impairment, preoperative Hoehn and Yahr stage and total UPDRS score, duration of Parkinson's disease, age of disease onset, duration of levodopa treatment, and preoperative doses of pergolide and bromocriptine. Patients who performed cognitively worse after pallidal stimulation were significantly older at the time of surgery (MannWhitney $U=17.5, \mathrm{p}=0.04$ ), and received higher preoperative doses of levodopa (MannWhitney $U=12.0, p=0.01)$. The mean age of

Table 3 Preoperative and postoperative neuropsychological raw test scores of patients with Parkinson's disease undergoing unilateral pallidal stimulation

\begin{tabular}{lllll}
\hline Cognitive measure & Preoperative score & Postoperative score & $F^{\star}$ & p Value \\
\hline AVLT immediate verbal recall & $43.3(9.6)$ & $42.7(10.8)$ & 0.04 & 0.837 \\
AVLT delayed verbal recall & $7.7(2.5)$ & $7.5(3.5)$ & 0.01 & 0.932 \\
BVRT visual recognition & $11.9(2.0)$ & $11.5(3.1)$ & 3.70 & 0.070 \\
PPT bimanually placed pegs & $12.4(4.0)$ & $11.8(5.0)$ & 0.56 & 0.465 \\
Visual reaction time right & $327.9(143.0)$ & $287.5(56.4)$ & 0.65 & 0.432 \\
Visual reaction time left & $380.0(384.4)$ & $309.0(90.0)$ & 0.82 & 0.377 \\
VOSP number location & $8.3(2.0)$ & $8.9(1.3)$ & 2.56 & 0.127 \\
VOSP cube analysis & $9.0(1.7)$ & $8.9(1.8)$ & 2.85 & 0.108 \\
MRMT left/right orientation & $29.2(2.8)$ & $30.0(1.9)$ & 1.61 & 0.220 \\
SCWT interference score & $69.2(37.9)$ & $64.7(42.4)$ & 0.40 & 0.535 \\
COWAT word fluency & $33.0(10.3)$ & $30.9(8.9)$ & 1.36 & 0.260 \\
WCST categories & $2.5(2.6)$ & $2.5(2.6)$ & 0.11 & 0.743 \\
\hline
\end{tabular}

Results are means(SD); *df always 1,18 . the cognitive decline group was 62 (SD 4) years, whereas the mean age of the no decline group was 52 (SD 10) years. Mean levodopa dose of the decline group measured 910 (SD 300) $\mathrm{mg}$, mean dose of the no decline group measured 660 (SD 240) mg. We found no significant correlation (Pearson) between age and levodopa dose.

We also found that the six patients showing cognitive decline all underwent deep brain stimulation of the left hemisphere $\left(\chi^{2}=4.62\right.$, $\mathrm{p}<0.05)$. We found no significant differences on the aforementioned demographic and medical variables between patients undergoing left hemispheric $(n=13)$ and right hemispheric $(n=7)$ deep brain stimulation.

A repeated measures MANOVA was performed with the 12 cognitive measures comparing the preoperative and postoperative group performance, using operation side (left/ right) as a between subjects factor. This showed no significant effect for time of assessment (main effect of operation; Hotelling's $T$ $(12,7)=1.33, p=0.665)$. The preoperative and postoperative raw scores for the total group on each neuropsychological measure and the results of the MANOVA post-hoc analyses using univariate $F$ tests are listed in table 3 . Inspection of these data disclosed only small differences between the preoperative and postoperative performances that were never significant. The side of stimulation did not reveal a significant differential effect on cognitive performance (main effect of lateralisation; Hotelling's $T(12,7)=3.42, \mathrm{p}=0.183)$. There was no significant operation by lateralisation interaction effect (Hotelling's $T \quad(12,7)=2.16$, $\mathrm{p}=0.392)$.

The mean preoperative depression score (cognitive/affective subscale) measured 7.8 (SD 6.4), range 0-21 (maximum 39). The mean postoperative depression score was 6.4 (SD 5.8), range 0-19 (maximum 39). The postoperative reduction was not statistically significant and both values indicate an average mild depressive symptomatology. We found no 
significant correlations (Pearson) between depression score, Hoehn and Yahr stage, UPDRS score, and cognitive impairment index before and after surgery.

\section{Discussion}

Our results suggest that no significant deterioration in cognitive status is seen after functional pallidotomy by means of unilateral pallidal stimulation. In general, the cognitive impairment in patients with Parkinson's disease that can be found after surgery is already present before the operation and neither significant postoperative decline in general cognitive impairment, nor specific cognitive test performance is seen when group data are compared. Compared with normal subjects of comparable sex, age, and level of education, the average patient with Parkinson's disease performs $<1$ SD below a normal performance in over one third of the cognitive tasks both before and after the neurosurgical intervention. The degree of cognitive impairment was not associated with the estimated intellectual level of the patient suggesting that higher IQ has neither a protective role against cognitive decline in Parkinson's disease, nor makes the patient more vulnerable for Parkinson's disease related neuropsychological impairment.

The absence of significant cognitive change after chronic pallidal stimulation in our study is in agreement with the results of some small sample pilot studies on the neuropsychological outcome after unilateral pallidotomy. ${ }^{75} 26 \mathrm{On}$ the other hand, cognitive deficits have been found in a larger group of patients with unilateral pallidotomy. ${ }^{27}{ }^{28}$ It can be argued that the evaluation of larger cohorts is necessary to appreciate group differences, ${ }^{27}$ although the methodological caveats regarding the comparison of means (mentioned in the methods section) also apply for larger group sizes. Analysing individual outcome, as was done in this study, can identify possible patient subgroups that are more vulnerable for neurosurgical intervention. Another consideration is the possible differential effect of pallidotomy through lesioning or electrostimulation. Only direct comparison in an identical methodological design can evaluate the differential neuropsychological impact of both surgical techniques.

Studies attempting to relate cognitive impairment to the asymmetry of motor symptoms in Parkinson's disease have found contradictory results. Some findings suggest that initial damage to right hemispheric dopaminergic systems is associated with more pervasive cognitive decline..$^{24}$ Other studies found no correlation between laterality of motor parkinsonian symptoms and cognitive asymmetry scores..$^{22}$ Because the patient groups undergoing left or right deep brain stimulation showed no significant differences on demographic and medical variables, the lateralisation variable was included as a between subjects factor in the repeated measures MANOVA. The results disclosed no significant effect of lateralisation, and no significant interaction effect. These findings suggest that neither asymmetry of motor
Parkinson's disease symptoms, nor subsequent unilateral stimulation has a differential effect on cognitive performance. The lack of influence of the side of stimulation could, however, be due to the few patients in each group. Examining a larger group, Lang et $a l^{27}$ found word fluency and verbal learning difficulties associated with left sided pallidotomy, and visuospatial dysfunction after right sided pallidotomy, although it remains unclear on which statistics these conclusions were based. Baron et $a l^{46}$ subsequently provided additional data on lateralisation effects after pallidotomy, but found no impact of the side of the GPi lesion on cognitive performance. Our finding that the six patients showing cognitive decline all underwent stimulation of the left hemisphere is noteworthy and warrants further investigation.

By contrast with the stability of the patients' cognitive status a significant postoperative improvement in Parkinson's disease symptomatology as reflected by neurological symptom scales was found. This finding confirmed previous reports focusing on postoperative changes in motor symptoms after pallidal stimulation. ${ }^{12-15}$ In general, the severity of motor symptomatology is not significantly related to the degree of cognitive impairment. Only after the operation is the UPDRS score moderately associated with the cognitive impairment index.

When we compared patients who had a cognitive decline after surgery (30\%) with patients who showed no change or even improvement in cognitive performance, it seemed that patients showing a cognitive decline were significantly older at the time of surgery and received higher preoperative doses of levodopa. Other major individual disease characteristics such as severity of preoperative motor symptoms, duration of Parkinson's disease, age of disease onset, and the duration of levodopa treatment showed no significant influence on cognitive outcome.

Finally, our results showed that in general patients with Parkinson's disease are mildly depressed before and after surgery. Depression scores were corrected for confounding vegetative and somatic symptoms in these medically ill patients. There was no significant reduction in self reported depression scores after surgery and depression scores were not significantly related to motor symptom scales and cognitive impairment. Depression is a frequent accompanying feature in patients with Parkinson's disease. ${ }^{17}{ }^{30}$ The exact aetiology of depression in Parkinson's disease (psychological reaction to the debilitating motor symptoms or concurrent structural and biochemical changes beyond those causing the motor syndrome) remains unclear. ${ }^{47}$ Our findings indicated no significant reduction in the reported feelings of depression in relation to the significant improvement in motor symptoms after surgery. These preliminary findings do not support evidence for a direct (psychological) relation between motor impairment and mood symptoms in Parkinson's disease.

Several methodological caveats of the current study deserve attention. Firstly, the results are based on a small sample of patients and the 
follow up period is brief. Whereas the results of the study in general suggest little risk of postoperative cognitive decline, longitudinal follow up with an increased sample size remains warranted. Secondly, the investigators were (for obvious reasons) not blind to the moment of assessment (before or after surgery) and examiner effects cannot therefore be excluded. Thirdly, the effects of repeated testing in a non-surgical control group were not evaluated. Even when alternative test versions for repeated testing are used the effect of task familiarity can improve test performance on subsequent cognitive assessments. The magnitude of the learning effect in Parkinson's disease candidates for deep brain stimulation is unknown. As a consequence, the interpretation of group data remains difficult. Analysis of individual preoperative to postoperative changes in cognitive performance can partially overcome this problem, but the question remains whether the absence of a learning effect (no preoperative to postoperative cognitive improvement) should be regarded as an indication of reduced cognitive abilities or should be considered normal for this population (as was done in this study by way of precaution). Fourthly, accurate placement of the lesion in the posterior GPi without damaging the overlying white matter and without impinging on associative circuits in the anterior GPi or the external pallidum (GPe) is a critical determinant for both motor and cognitive outcomes. ${ }^{46}$ Even with the use of microelectrode recording techniques to guide lesion placement, the exact location of the lesion is difficult to confirm. This is even more so with electrostimulation because the exact size of the functional lesion cannot be visualised. Some of our lesions might, therefore, impinge on adjacent brain regions. Fifthly, because of the difficulties in testing patients with advanced Parkinson's disease, and the fact that most of these patients are easily fatigued, the neuropsychological test battery had to be limited. As we mentioned in the methods section, we focused on those cognitive domains thought to be affected in Parkinson's disease instead of covering a wider range of cognitive domains that might have disclosed other postoperative changes. Given the uncertainties about the accuracy of lesion placement, and given the limitations of the test battery, we cannot exclude the existence of unidentified postoperative cognitive deficits.

We conclude that unilateral pallidal stimulation, regardless of stimulation side, seems relatively safe, although older patients and patients taking high preoperative levodopa doses seem to be more vulnerable for postoperative cognitive decline. Future, preferably longitudinal, research on a larger sample of patients is warranted. Comparing not only groups of patients but also examining the changes in individual performance over time may further elucidate the effect of chronic pallidal stimulation on cognitive function.

1 Aminoff MJ. Treatment of Parkinson's disease. West $\mathcal{F}$ Med 1994;161:303-8.
2 Marsden CD, Parkes JD. Succes and problems of long-term

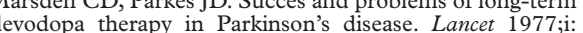
levodopa

345-9

Nutt JG. On-off phenomenon: relation to levodopa pharmacokinetics and pharmacodynamics. Ann Neurol 1987;22:535-40

4 Dogali M, Fazzani E, Kolodny E, et al. Stereotactic ventral pallidotomy for Parkinson's disease. Neurology 1995;45: 753-61.

5 Iacono RP, Lonser RR, Oh A, et al. New pathophysiology of Parkinson's disease revealed by posteroventral pallidotomy. Neurol Res 1995;17:178-80.

6 Lozano AM, Lang AE, Galvez-Jimenez N, et al. Effect of GPi pallidotomy on motor function in Parkinson's disease. Lancet 1995;346:1383-7.

7 Baron MS, Vitek JL, Bakay RAE, et al. Treatment of advanced Parkinson's disease by posterior GPi pallidotomy: 1-year results of a pilot study. Ann Neurol pallidotomy: 1-year

8 Laitinen LV. Ventroposterolateral pallidotomy. Stereotact Funct Neurosurg 1994;62:41-52.

9 Eidelberg D, Moeller JR, Ishikawa T, et al. Regional metabolic correlates of surgical outcome following unilateral pallidotomy for Parkinson's disease. Ann Neurol 1996; 39:450-9

10 Grafton ST, Waters C, Sutton J, et al. Pallidotomy increases activity of motor association cortex in Parkinson's disease: a positron emission tomographic study. Ann Neurol 1995;37:776-83.

11 Laitinen LV, Bergenheim AT, Hariz MI. Ventroposterolateral pallidotomy can abolish all parkinsonian symptoms. Stereotact Funct Neurosurg 1992;58:14-21.

12 Iacono RP, Lonser RR, Maeda G, et al. Chronic anterior pallidal stimulation for Parkinson's disease. Acta Neurochir Wien 1995;137:106-12.

13 Iacono RP, Lonser RR, Mandybur G, et al. Stimulation of the globus pallidus in Parkinson's disease. $\mathrm{Br} \mathcal{7}$ Neurosurg 1995;9:505-10

14 Siegfried J, Lippitz B. Bilateral chronic electrostimulation of ventroposterolateral pallidum: a new therapeutic approach for alleviating all parkinsonian symptoms. Neurosurgery 1994;35:1126-9.

15 van der Linden Ch, Caemaert J, Vandewalle V, et al. Chronic unilateral stimulation of the internal globus pallidus (Gpi) in advanced Parkinson's disease (PD): a 3 month follow up in nine patients. Neurology 1997;48:A430.

16 Dogali M, Beric A, Sterio D, et al. Anatomic and physiological considerations in pallidotomy for Parkinson's disease. Stereotact Funct Neurosurg 1994;62:53-60.

17 Saint-Cyr JA, Taylor AE, Nicholson K. Behavior and the basal ganglia. In: Weiner WJ, Lang AE, eds. Behavioral neurology of movement disorders, advances in neurology. Vol 65 . rology of movement disorders, advances

18 Limousin P, Greene J, Pollak P, et al. Canges in cerebral activity pattern due to subthalamic nucleus or internal pallidum stimulation in Parkinson's disease. Ann Neurol 1997; 42:283-92.

19 Sabatino M, La Grutta V, Ferraro G, et al. Relations between basal ganglia and hippocampus: action of substantia nigra and pallidum. Rev Electroencephalogr Neurophysiol Clin 1986;16:179-90.

20 Sabatino M, Savatteri V, Liberti G, et al. Effects of substantia nigra and pallidum stimulation on hippocampal interictal activity in the cat. Neurosci Lett 1986;64:293-8.

21 Tranel D, Damasio AR. Neurobiological foundations of human memory. In Baddeley AD, Wilson BA, Watts FN, eds. Handbook of memory disorders. Chichester: Wiley, 1995: 27-50.

22 Agniel A, Celsis P, Viallard G, et al. Cognition and cerebral blood flow in lateralised Parkinsonism: lack of functional lateral asymmetries. F Neurol Neurosurg Psychiatry 1991;54: 783-6.

23 Mohr E, Mann UM, Miletich RS, et al. Neuropsychological and glucose metabolic profiles in asymmetric Parkinson's disease. Can $\mathcal{F}$ Neurol Sci 1992;19:163-9.

24 Tomer R, Levin BE, Weiner WJ. Side of onset of motor symptoms influences cognition in Parkinson's disease. Ann Neurol 1993;34:579-84.

25 Green J, Vitek JL, Baron M, et al. Neuropsychological sequelae of pallidotomy for treatment of Parkinson's disease: oneyear pilot findings. Neurology 1996;48(suppl):A200.

26 Soukop VM, Ingram F, Schiess MC, et al. Cognitive sequelae of unilateral posteroventral pallidotomy. Arch Neurol 1997;54:947-50.

27 Lang AE, LOzano A, Tasker R, et al. Neuropsychological and behavioral changes and weight gain after medial palliand behavioral changes and weight

28 Saint-Cyr JA, Trépanier LL, Lang AE, et al. Neuropsychological outcome of posteroventral pallidotomy in parkinsonian patients. Mov Disord 1996;11(suppl):161.

29 Dubois B, Pillon B. Cognitive deficits in Parkinson's disease. f Neurol 1997;244:2-8.

30 Levin BE, Katzen HL. Early cognitive changes and non-dementing behavioral abnormalities in Parkinson's disease. In: Weiner WJ, Lang AE, eds. Behavioral neurology of movement disorders, advances in neurology. Vol 65. New York: Raven Press, 1995:85-95.

31 Lezak MD. Neuropsychological assessment. 3rd ed. New York: Oxford University Press, 1995.

32 Benton AL. Der Benton Test. Handbuch. (The Benton test. Manual.) Bern: Verlag Hans Huber, 1961.

33 Fepsy. The iron psyche. Manual. Heemstede, The Fepsy. The iron psyche. Manual. Heemstede, The jding, 1995. 
34 Warrington EK, Merle J. Visuele Perceptie van Object en Ruimte. Handleiding. (The visual object and space perception battery. Manual)

35 Hammes J. Stroop Kleur-Woord Test: Handleiding (Manual of the Stroop colour-word test). Amsterdam: Swets and Zeitlinger, 1971.

36 Heaton RK, Chelune GJ, Talley JL, et al. Wisconsin card sorting test. Manual-revised and expanded. Odessa, Fl: Psychological Assessment Recources, 1993. 37 Schmand B, Lindeboom J, van Harskamp F. Nederlandse
Leestest voor Volwassenen (Dutch adult reading test). Lisse, The Netherlands: Swets and Zeitlinger BV, 1992.

38 Beck AT, Steer RA. Beck depression inventory. Manual. San Antonio: The Psychological Corporation, 1993.

39 Clark DA, Steer RA. Use of non-somatic symptoms to differentiate clinically depressed and non-depressed hospitalized patients with chronic medical illnesses. Psychol Rep 1994;75:1089-90.

40 Plumb MM, Holland J. Comparative studies of psychological function in patients with advanced cancer, I: self-reported depressive symptoms. Psychosom Med 1977;39:264-79.
41 Vingerhoets G. Perioperative anxiety and depression in open-heart surgery. Psychosomatics 1998;39:30-7.

42 Parkinson Study Group. Effect of deprenyl on the progression of disability in early Parkinson's disease. N Engl f Med 1989;328:176-83.

43 Fahn S, Elton R, Members of UPDRS Development Committee. In: Fahn S, Marsden CD, Caine DB, et al, eds. Recent developments in Parkinson's disease. Vol 2. Florham Park, NJ: Macmillan Health Care Information, 1987.

44 Fepsy. The iron psyche. Normative data. Vols 2 and 3. Heemstede, The Netherlands: Nederlands Instituut voor Epilepsiebestrijding, 1995.

45 Lannoo E, Vingerhoets G. Influence of age, education and gender on neuropsychological test performance of normal healthy Flemish adults. Psychol Belg 1997;37:141-56.

46 Baron MS, Vitek JL, Bakay RAE, et al. Reply to Lang et al. Ann Neurol 1997;41:835-6.

47 Stern Y, Mayeux R. Mental dysfunction in Parkinson's disease. In: Wolters EC, Scheltens P, eds. Mental dysfunction in Parkinson's disease. Amsterdam: Vrije Universiteit, 1993: 123-32. 